Discrete Comput Geom 28:607-624 (2002)

DOI: $10.1007 / \mathrm{s} 00454-002-2892-3$

Geometry

\title{
Gallai-Sylvester Theorem for Pairwise Intersecting Unit Circles
}

\author{
Rom Pinchasi \\ Institute of Mathematics, Hebrew University of Jerusalem, \\ Givat Ram, Jerusalem, Israel \\ room@math.huji.ac.il
}

\begin{abstract}
For every finite family of (at least five) pairwise intersecting unit circles in the plane, there is an intersection point that lies on exactly two circles. This proves a conjecture of A. Bezdek.
\end{abstract}

\section{Introduction}

The celebrated Gallai-Sylvester theorem ([S], $[\mathrm{G}]$, and see also [BM], [M], and [PA] for a survey of the history and generalizations of this theorem), in its dual form, asserts that for every finite collection of lines in the real projective plane that do not form a pencil (i.e., do not share a common point) there is a point that lies on exactly two lines. A possible analogue for circles could be: in any finite collection of (at least two) unit circles that are pairwise intersecting (i.e., every two circles meet) there is an intersection point that belongs to exactly two circles.

Unfortunately, this statement is false. A simple counterexample consists of three distinct unit circles through a common point $P$, each two meeting in two points, and a fourth unit circle that passes through the remaining three intersection points. We call this family exceptional (see Fig. 1). Bezdek [B1] conjectured that this is the only counterexample to the statement above. (In [B1] Bezdek actually refers to the dual statement, namely, for any set of $n$ points in the plane with diameter $\leq 2$ there exists a unit circle which passes through precisely two points. See [BB] for some closely related results about circles determined by a small number of points.) He confirmed this under the additional condition that the distance between the centers of any two unit circles is less than $\sqrt{3}$. Bezdek used this condition to bound the number of lunes and lenses (definitions will follow) induced by the family of unit circles. This bound (which is actually four) and Euler formula give the desired result.

In this paper we prove Bezdek's conjecture, namely, we prove the following theorem. 


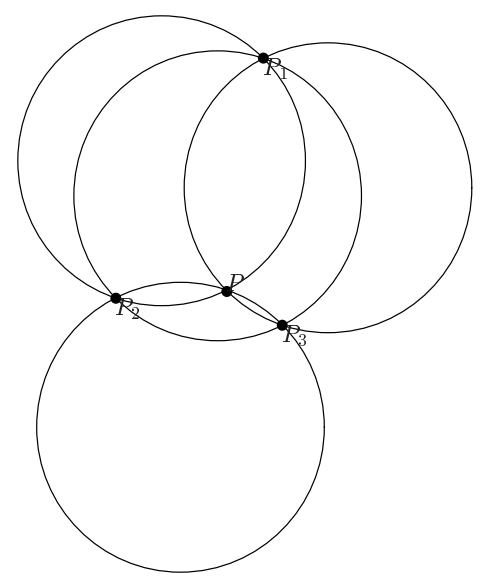

Fig. 1. An exceptional configuration.

Theorem 1.1. Let $\mathcal{C}$ be a finite family of (at least two) pairwise intersecting unit circles in the plane. Then there is a point through which exactly two circles pass, unless $\mathcal{C}$ is exceptional.

Remark. Under the same assumptions of Theorem 1.1, one can show rather easily that there exists an intersection point through which few circles pass (consult [BFT] for a short argument proving the existence of an intersection point incident to at most five unit circles). Even under weaker assumptions than in Theorem 1.1 one can show the existence of an intersection point incident to at most three circles [ALPS], [NPPS], as described in more detail in the concluding remarks section. Proving Theorem 1.1 requires a more delicate argumentation. One of the reasons for this is, apparently, the existence of a small counterexample to this theorem, namely the exceptional configuration.

There are several ways in which one can try to generalize Theorem 1.1. Consider first the case where $\mathcal{C}$ is a family of circles of arbitrary radii which are pairwise intersecting. There is an obvious counterexample to this generalization, simply take $\mathcal{C}$ to be a pencil of circles, that is, circles which pass through two given points. Then there are only two intersection points and each is incident to all circles in $\mathcal{C}$. We can impose natural restrictions on the family $\mathcal{C}$, such as that $\mathcal{C}$ is not a pencil, or that at most two circles pass through any two given points (as in the case of unit circles).

In each of those two cases we have a small counterexample to Theorem 1.1, other than the one given in Fig. 1. This example is given by Fig. 2 .

There are six circles in the family $\mathcal{C}$ and a dotted one. The family of the six full circles does not have an intersection point of precisely two circles, and satisfies the condition that at most two circles pass through any two given points. If we add the dotted circle we overrule this condition but still $\mathcal{C}$ is not a pencil, and again there is no intersection point through which exactly two circles pass.

Finally, note that if the family $\mathcal{C}$ consists of unit circles which are not necessarily pairwise intersecting, then the analogue of Theorem 1.1 to this case is not valid. This is clearly true if $\mathcal{C}$ is a family of disjoint unit circles. If we insist that $\mathcal{C}$ be a connected 


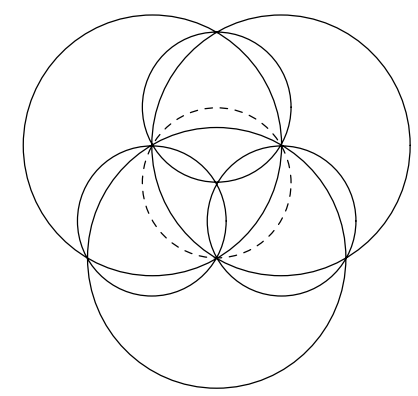

Fig. 2. An exceptional configuration.

family, then one can build a counterexample by extending the example given in Fig. 1. Namely, assume we already have a configuration $\mathcal{C}$ of $n \geq 1$ unit circles with no simple intersection point. Let $Q$ be a point, on a circle $C \in \mathcal{C}$, which is also an extreme point of the convex hull of $\cup \mathcal{C}$. Choose three points $P_{1}, P_{2}, P_{3}$ on $C$, very close to each other and very close to $Q$. There are exactly three unit circles different from $C$ which pass through exactly two of the points $P_{1}, P_{2}, P_{3}$. These three unit circles meet at a common point $P$ which is "far" from the original configuration. Adjoining these three unit circles to $\mathcal{C}$ will now produce a new configuration of $n+3$ unit circles with no simple intersection point.

\section{Definitions and Notation}

Throughout this paper $\mathcal{C}$ denotes a finite family of at least three (clearly, Theorem 1.1 is valid when $\mathcal{C}$ consists of just two circles) unit circles in the Euclidean plane. We assume that every two (distinct) circles in $\mathcal{C}$ meet. Every two circles in $\mathcal{C}$ either cross (i.e., have two points in common) or touch (i.e., have a unique point in common). We denote by $\mathcal{A}(\mathcal{C})$ the planar map induced by $\mathcal{C}$. Its vertices $\mathcal{V}(\mathcal{C})$ are the intersection points of circles in $\mathcal{C}$, its edges are the closures of the components of $\cup \mathcal{C} \backslash \mathcal{V}(\mathcal{C})$, and its faces are the closures of the components of $\mathrm{R}^{2} \backslash \cup \mathcal{C}$. We denote the (planar) graph of $\mathcal{A}(\mathcal{C})$ by $G_{\mathcal{C}}$. $G_{\mathcal{C}}$ may have multiple edges, but no loops (unless $\mathcal{C}$ consists of just two touching circles, a case which we excluded).

We usually denote a unit circle by $C$, possibly with some modifier (subscript or superscript). The closed disk bounded by that circle is denoted by $D$, and its center by $O$, with the same modifier.

For a circle $C$ and points $A, B$ on $C$ that are not antipodal, we denote by $\operatorname{arc}_{C}(A B)$ the closed shortest arc on $C$ between $A$ and $B$.

For two distinct points $A$ and $B$ in the plane we denote by $\overline{A B}$ the line through $A$ and $B$. We denote by $\overrightarrow{A B}$ the closed ray which starts at $A$ and includes $B$. [AB] denotes the closed interval between $A$ and $B$.

For every non-collinear point $P, Q, R$, we denote by $\triangle(P Q R)$ the closed triangle with vertices $P, Q$, and $R . \angle P Q R$ denotes the closed convex region bounded by the rays $\overrightarrow{Q P}$ and $\overrightarrow{Q R}$. $\measuredangle P Q R$ denotes the angular measure of $\angle P Q R$. Therefore, $0<\measuredangle P Q R<\pi$. 
Definition 2.1. The degree $d(P)$ of a point $P \in \mathcal{V}(\mathcal{C})$ is the number of circles in $\mathcal{C}$ that pass through $P$. We call $P$ a simple intersection point when $d(P)=2$.

For $k \geq 2$ denote by $t_{k}$ the number of intersection points of degree $k$. The number $V$ of vertices is $\sum_{k \geq 2} t_{k}$. Denote by $f_{k}$ the number of faces of $G_{\mathcal{C}}$ which have $k$ edges (we also regard the unbounded face). The number $F$ of faces is $\sum_{k \geq 2} f_{k}$. Denote by $E$ the number of edges of $G_{\mathcal{C}}$. We have

$$
2 E=\sum_{k \geq 2} k f_{k} \quad \text { as well as } \quad E=\sum_{k \geq 2} k t_{k} .
$$

Therefore

$$
2 E=\sum_{k \geq 2} k f_{k}=3 F+\sum_{k \geq 2}(k-3) f_{k} .
$$

Clearly, $G_{\mathcal{C}}$ is connected and has no loops. Hence, by Euler's formula, $V-E+F=2$. Therefore,

$$
\begin{aligned}
-6 & =-3 V+3 E-3 F=-3 V+E+(2 E-3 F) \\
& =-3 \sum_{k \geq 2} t_{k}+\sum_{k \geq 2} k t_{k}+\sum_{k \geq 2}(k-3) f_{k} \\
& =\sum_{k \geq 2}(k-3) t_{k}+\sum_{k \geq 2}(k-3) f_{k} .
\end{aligned}
$$

Rearranging, we get

$$
t_{2}=6+\sum_{k \geq 3}(k-3) t_{k}+\sum_{k \geq 3}(k-3) f_{k}-f_{2} .
$$

According to this notation Theorem 1.1 asserts that $t_{2}>0$ (unless $\mathcal{C}$ is exceptional). As is easily seen from (1), this will follow if we show that $f_{2}$ is "small" relative to the other terms on the right-hand side of the equation. To this end we introduce the notion of lunes and lenses.

Definition 2.2. Let $C_{1}, C_{2}$ be two circles in $\mathcal{C}$. In case $C_{1}$ and $C_{2}$ cross, we call $L=$ $D_{1} \cap D_{2}$ a lens if it is a face with two edges in the planar graph $G_{\mathcal{C}}$. We then say that both $C_{1}$ and $C_{2}$ support $L$ (see Fig. 3). The two intersection points of $C_{1}$ and $C_{2}$ are called the vertices of $L$. If $C_{1}$ and $C_{2}$ touch at $P$, we call $P$ a lens if $d(P) \leq 3$. Also in this case we say that $C_{1}$ and $C_{2}$ support $P$. A lens that is not a single point is called a proper lens.

We call $D_{1} \backslash$ int $D_{2}$ a lune if it is a face with two edges of the planar graph $G_{\mathcal{C}}$. We then call $C_{1} \backslash$ int $D_{2}$ the longest arc of the lune and say that $C_{1}$ supports the longest arc of that lune.

Observation 2.3. The length of the longest arc of a lune is greater than $\pi$.

Clearly, every two-edge face is either a lune or a proper lens. In the next section we bound the number of lunes and lenses. We show (Lemma 3.4) that the number of lenses 


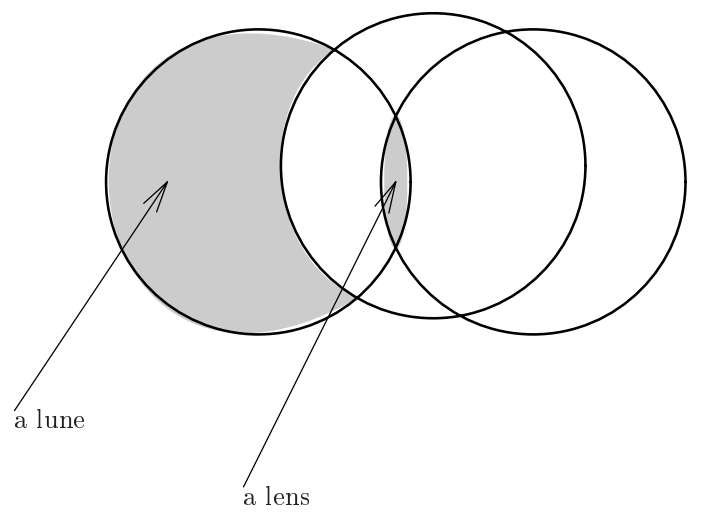

Fig. 3. A lens and a lune.

in any configuration of $n$ pairwise intersecting unit circles is at most $n$. In Lemma 3.2 we show that if $C$ supports a lens, then it must contain an edge of the unbounded face of $\mathcal{A}(\mathcal{C})$. As a corollary of these two results we conclude (at the beginning of the proof of Theorem 1.1) that the number of lenses is less than or equal to the number of edges of the unbounded face of $\mathcal{A}(\mathcal{C})$. This is a crucial step towards showing that the right-hand side of (1) is positive. The lunes seem to make much less trouble. In Corollary 3.10 we bound the number of lunes by three. Section 4 completes the proof.

\section{Some Lemmata}

\section{Lemma 3.1.}

(i) Let $C_{1}, C_{2}, C_{3} \in \mathcal{C}$ such that $D_{1} \cap D_{2} \cap D_{3}$ is at most one point. Then $\triangle\left(O_{1} O_{2} O_{3}\right)$ is acute unless two of the circles touch and $D_{1} \cap D_{2} \cap D_{3} \neq \emptyset$.

(ii) Assume $C, C_{1}, C_{2} \in \mathcal{C}$. If $D \cap D_{1}$ and $D \cap D_{2}$ are distinct lenses, then all angles of $\Delta\left(O O_{1} O_{2}\right)$ are $\leq \pi / 2$.

Proof. (i) We show that the angle at the vertex $\mathrm{O}_{3}$ of $\Delta\left(\mathrm{O}_{1} \mathrm{O}_{2} \mathrm{O}_{3}\right)$ is acute unless $\mathrm{C}_{1}$ and $C_{2}$ touch at a point $P$, in which case $\measuredangle O_{1} O_{3} O_{2}<\pi / 2$ iff $P \notin D_{3}$. The same argument will apply of course to the angles at $O_{1}$ and $O_{2}$.

If $C_{1}$ and $C_{2}$ touch at a point $P$, then $\measuredangle O_{1} O_{3} O_{2}<\pi / 2$ iff the distance from $P$ to $O_{3}$ is $>1$ or in other words iff $P \notin D_{3}$.

If $C_{1}$ and $C_{2}$ cross, denote by $P$ the midpoint of $\left[O_{1} O_{2}\right] . P \in \operatorname{int} D_{1} \cap$ int $D_{2}$, and $\measuredangle O_{1} O_{3} O_{2} \geq \pi / 2$ would imply that $P \in$ int $D_{3}$ as well. This is impossible since $\operatorname{int}\left(D_{1} \cap D_{2} \cap D_{3}\right)=\emptyset$.

Part (ii) follows immediately from (i), if no two of the circles $C, C_{1}, C_{2}$ touch or if $D_{1} \cap D_{2} \cap D_{3}=\emptyset$. Assume therefore that $D_{1} \cap D_{2} \cap D_{3} \neq \emptyset$, and that two of the circles touch at $P$. If the third circle passes through $P$, then $\Delta\left(O O_{1} O_{2}\right)$ is a right triangle. If the third circle does not pass through $P$, then it must contain $P$ in its interior. Consequently, this circle cannot create a lens with any of the two touching circles, a contradiction. 


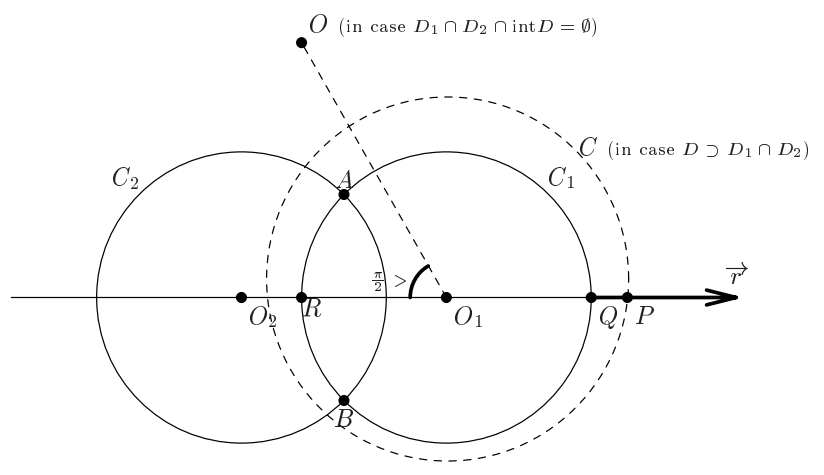

Fig. 4. Lemma 3.2.

Lemma 3.2. Assume $D_{1} \cap D_{2}$ is a lens with vertices $A, B$ (possibly $A=B$ ), and denote by $Q$ the intersection point of $\overrightarrow{O_{2} O_{1}}$ with $C_{1}$. Then

1. $C_{1}$ contains an edge of the unbounded face of $\mathcal{A}(\mathcal{C})$.

2. If $C \in \mathcal{C}$ and int $D$ is disjoint from $D_{1} \cap D_{2}$, then the arc $C_{1} \cap D$ is included either in $\operatorname{arc}_{C_{1}}(Q A) \backslash\{Q\}$ or in $\operatorname{arc}_{C_{1}}(Q B) \backslash\{Q\}$. (If $A=B$ then by $\operatorname{arc}_{C_{1}}(Q A)$ and $\operatorname{arc}_{C_{1}}(Q B)$, we mean the two semicircles of $C_{1}$ from both sides of $[A, Q]$.)

Proof. 1. Let $\vec{r} \subset \overline{O_{1} O_{2}}$ be the ray which starts at $Q$ and does not include $O_{1}$ (see Fig. 4). It is enough to show that $\vec{r}$ does not meet any circle of $\mathcal{C}$ but $C_{1}$.

Assume $C \in \mathcal{C} \backslash\left\{C_{1}\right\}$ meets $\vec{r}$ at a point $P . D_{1} \cap D_{2}$ is a lens, and therefore either $D \supset D_{1} \cap D_{2}$ or $\left(D_{1} \cap D_{2}\right) \cap$ int $D=\emptyset$.

If $D \supset D_{1} \cap D_{2}$, then in particular $D$ includes the point $R \in C_{1}$ that lies opposite to $Q$ (see Fig. 4). It follows (since $P, R \in C \neq C_{1}$ ) that the diameter of $D$ is then greater than two, which is impossible.

If, on the other hand, $\left(D_{1} \cap D_{2}\right) \cap$ int $D=\emptyset$, then, within the family $\mathcal{C}^{\prime}=\left\{C_{1}, C_{2}, C\right\}$, $D \cap D_{2}$ is also a lens. By Lemma 3.1, $\measuredangle O_{2} O_{1} O \leq \pi / 2$ (see Fig. 4). This implies that the distance between $P$ and $O$ is greater than 1, i.e., $P \notin C$.

2. Let $a$ denote the $\operatorname{arc} C_{1} \cap D$ of the circle $C_{1}$. Since we assume int $D \cap\left(D_{1} \cap D_{2}\right)=\emptyset$, $\operatorname{arc}_{C_{1}}(A B) \cap$ int $D=\emptyset$. Moreover, by the first part of Lemma 3.2 $Q \notin D$. It follows that $a$ is included either in $\operatorname{arc}_{C_{1}}(Q A)$ or in $\operatorname{arc}_{C_{1}}(Q B)$.

Our next goal is to prove Lemma 3.4 which says that in an arrangement of $n$ pairwise intersecting unit circles there are at most $n$ lenses. We shall need the following auxiliary lemma.

Lemma 3.3. Assume $C_{1}, C_{2}, C_{3}, C_{4} \in \mathcal{C}$ are four distinct circles. If the four intersections $D_{1} \cap D_{3}, D_{1} \cap D_{4}, D_{2} \cap D_{3}$, and $D_{2} \cap D_{4}$ are lenses, then $C_{1}$ and $C_{2}$ touch at a point $P$, and $C_{3}$ and $C_{4}$ touch at $P$ as well.

Proof. We restrict our attention to the subfamily $\mathcal{C}^{\prime}=\left\{C_{1}, C_{2}, C_{3}, C_{4}\right\}$. Note that if the intersection $D_{i} \cap D_{j}$ is not a lens in $\mathcal{C}^{\prime}$, then it is not a lens in $\mathcal{C}$ as well. By Lemma 3.1, all 
12 angles $\angle O_{i} O_{j} O_{k}(i, j, k \in\{1,2,3,4\})$ are less than or equal to $\pi / 2$. However, this is possible only if $O_{1}, O_{2}, O_{3}, O_{4}$ are the vertices of a rectangle $R$. Let $P$ the the center of $R$. The four intervals [ $P, O_{i}$ ] $, i=1,2,3,4$, all have the same length $d$. If $d<1$, then $P \in \operatorname{int}\left(D_{1} \cap D_{2} \cap D_{3} \cap D_{4}\right)$. This is impossible since already int $\left(D_{1} \cap D_{2} \cap D_{3}\right)=\emptyset$, as $D_{1} \cap D_{3}$ and $D_{2} \cap D_{3}$ are lenses. If $d>1$, then the circles centered at opposite vertices of $R$ are disjoint. There remains the case $d=1$. In this case all four circles pass through $P$, and the two circles centered at opposite vertices of $R$ touch at $P . P$ is not a lens, because it lies on four circles. It follows that the vertex of $R$ opposite to $O_{1}$ is $O_{2}$, and $\mathrm{O}_{4}$ lies opposite to $\mathrm{O}_{3}$.

Lemma 3.4. If $\#(\mathcal{C})=n$, then there are at most $n$ lenses in $\mathcal{A}(\mathcal{C})$.

Proof. By induction on $n$. The statement is clearly true for $n=2,3$. If there is a circle $C \in \mathcal{C}$ which supports at most one lens, then take $\mathcal{C}^{\prime}=\mathcal{C} \backslash\{C\}$. By the induction hypothesis, $\mathcal{C}^{\prime}$ has at most $n-1$ lenses. Adding $C$ to $\mathcal{C}^{\prime}$, will produce at most one more lens (and could possibly kill a few lenses of $\mathcal{A}\left(\mathcal{C}^{\prime}\right)$ ).

If every circle in $\mathcal{C}$ supports exactly two lenses, then the number of lenses is $n$, since every lens is supported by exactly two circles.

The proof of Lemma 3.4 will follow now from the following lemma which asserts that if a circle $C \in \mathcal{C}$ supports three lenses, then there is another circle in $\mathcal{C}$ which supports just one lens.

Lemma 3.5. Let $C, C_{1}, C_{2}, C_{3} \in \mathcal{C}$ be four distinct circles. Assume that $D \cap D_{i}(i=$ $1,2,3)$ are lenses. Then for some $j \in\{1,2,3\}, C_{j}$ supports no lens other than $D \cap D_{j}$.

Proof. By Lemma $3.1 \measuredangle O_{i} O O_{j} \leq \pi / 2$ for $1 \leq i<j \leq 3$. Assume, without loss of generality, that $O_{2} \in \operatorname{int} \angle O_{1} O O_{3}$. Rotate (and if necessary flip) the plane so that the line $\overline{O O_{2}}$ is vertical, with $O_{2}$ above $O, O_{1}$ is to the left of $\overline{O O_{2}}$, and $O_{3}$ is to the right of $\overline{\mathrm{OO}_{2}}$.

Let $Q=\overrightarrow{\mathrm{OO}_{2}} \cap C_{2}$ and $P=\overrightarrow{\mathrm{O}_{2} O} \cap C$. Denote the intersection points of $C$ and $C_{2}$ by $A, B$. If $D \cap D_{2}$ is not a proper lens, then $A=B \in \overline{O O_{2}}$. Otherwise, assume that $A$ is to the left of $\overline{O O_{2}}$. In what follows, when $A=B \operatorname{arc}_{C_{2}}(A Q)$ denotes the semicircle of $C_{2}$ which is to the left of $\overline{O_{2}}$, and $\operatorname{arc}_{C_{2}}(B Q)$ denotes the semicircle of $C_{2}$ which is to the right of $\overline{\mathrm{OO}_{2}}$.

We show that $C_{2}$ supports no lens other than $D \cap D_{2}$. Assume to the contrary, that $C_{0} \in \mathcal{C}, C_{0} \neq C$, and $D_{0} \cap D_{2}$ is a lens.

By Lemma 3.1, $\triangle\left(\mathrm{OO}_{0} \mathrm{O}_{2}\right)$ is a proper triangle, hence $\mathrm{O}_{0} \notin \overline{\mathrm{OO}_{2}}$. Assume, without loss of generality, that $\mathrm{O}_{0}$ is to the right of $\overline{\mathrm{OO}_{2}}$. We restrict our attention to the subfamily $\mathcal{C}^{\prime}=\left\{C, C_{0}, C_{1}, C_{2}\right\}$ and intend to show that $D_{0} \cap D_{1}$ is a lens in $\mathcal{C}^{\prime}$, contradicting Lemma 3.3. (Indeed, by Lemma 3.3, this is possible only if $C$ and $C_{0}$ touch at a point $V$, and $C_{1}, C_{2}$ touch at $V$ as well. This is impossible, since $O_{1}$ is to the left of $\overline{O O_{2}}$ and $\mathrm{O}_{0}$ is to the right of $\overline{\mathrm{OO}_{2}}$.)

Next come four applications of Lemma 3.2. With $C_{0}, C_{2}, C$ standing for $C, C_{1}, C_{2}$, respectively, and since $\mathrm{O}_{0}$ is to the right of $\overline{\mathrm{OO}_{2}}$, we have that

$$
C_{2} \cap D_{0} \subseteq \operatorname{arc}_{C_{2}}(Q B) \backslash\{Q\} .
$$


Similarly,

$$
\begin{array}{ll}
\text { with } C_{0}, C, C_{2} \text { we get } & C \cap D_{0} \subseteq \operatorname{arc}_{C}(P B) \backslash\{P\}, \\
\text { with } C_{1}, C_{2}, C \text { we get } & C_{2} \cap D_{1} \subseteq \operatorname{arc}_{C_{2}}(Q A) \backslash\{Q\}, \\
\text { with } C_{1}, C, C_{2} \text { we get } & C \cap D_{1} \subseteq \operatorname{arc}_{C}(P A) \backslash\{P\} .
\end{array}
$$

Therefore,

$$
C \cap D_{0} \cap D_{1}, C_{2} \cap D_{0} \cap D_{1} \subseteq\left\{\begin{array}{lll}
\varnothing & \text { if } \quad A \neq B, \\
\{A\} & \text { if } \quad A=B .
\end{array}\right.
$$

If $A=B$, then $\{A\}$ is a lens and so $A \notin C_{0} \cap C_{1}$. Thus if $A \in D_{0} \cap D_{1}$, then $A$ is an interior point of $D_{0}$ or $D_{1}$, but this is not possible because $D_{2} \cap D_{0}$ and $D \cap D_{1}$ are both lenses. Therefore $C \cap D_{0} \cap D_{1}$ and $C_{2} \cap D_{0} \cap D_{1}$ are always empty which proves that $D_{0} \cap D_{1}$ is a lens in $\mathcal{C}^{\prime}$.

Our next aim is to study the lunes in $\mathcal{A}(\mathcal{C})$, and more specifically the relationship between lunes and lenses in $\mathcal{A}(\mathcal{C})$.

We denote the length of a circular arc $S$ by $|S|$.

Lemma 3.6. Let $C, C^{\prime}$ be distinct circles in $\mathcal{C}$, with $C \cap C^{\prime}=\{A, B\}$ (possibly $A=B$, if $C$ and $C^{\prime}$ touch). Assume $\left|\operatorname{arc}_{C}(A B)\right|=\left|\operatorname{arc}_{C^{\prime}}(A B)\right|=\alpha$. Denote by $L, L^{\prime}$ the rays tangent to $\operatorname{arc}_{C}(A B)$ and $\operatorname{arc}_{C^{\prime}}(A B)$ at $A$. Then

1. The angle between $L$ and $L^{\prime}$ is $\alpha$.

2. The intersection of $C$ (or $\left.C^{\prime}\right)$ with the convex angular region bounded by $L$ and $L^{\prime}$ has length $2 \alpha$.

Proof. See Fig. 5. Note that the ray $\overrightarrow{A B}$ makes an angle $\frac{1}{2} \alpha$ with each of the tangents $L, L^{\prime}$.

Lemma 3.7. Suppose $C \in \mathcal{C}$ supports the longest arc of a lune. Then every lens that is included in $D$ must be supported by $C$. Moreover, if $C_{1}$ and $C_{2}$ are two circles which touch at a point $P \in D$, then either $C=C_{1}$ or $C=C_{2}$.

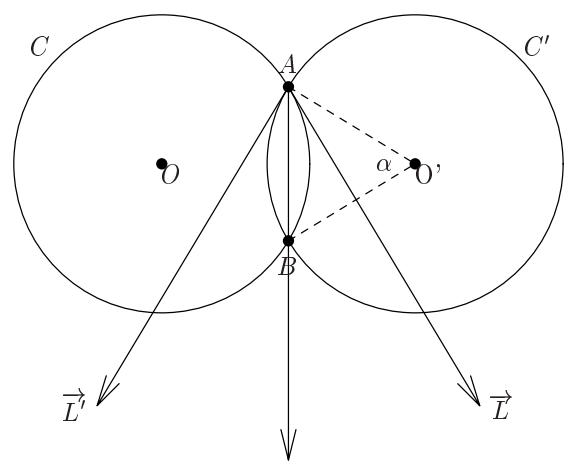

Fig. 5. Lemma 3.6. 
Proof. It is enough to prove that if $C_{1}, C_{2} \in \mathcal{C} \backslash\{C\}$, then $D_{1} \cap D_{2} \nsubseteq \nsubseteq D$.

Suppose $D \backslash$ int $D^{\prime}$ is a lune. Then the $\operatorname{arc} C \cap D^{\prime}$ is included in an open semicircle $S$, and for any circle $C_{i} \in \mathcal{C} \backslash\{C\}$ we have $C \cap D_{i} \subset C \cap D^{\prime} \subset S$. Assume first that $C \cap D_{1}$ and $C \cap D_{2}$ do not have interior points in common.

Let $C \cap C_{1}=\left\{A^{\prime}, B^{\prime}\right\}$ (possibly $\left.A^{\prime}=B^{\prime}\right)$ and $C \cap C_{2}=\left\{A^{\prime \prime}, B^{\prime \prime}\right\}\left(\right.$ possibly $\left.A^{\prime \prime}=B^{\prime \prime}\right)$, and assume that the points $A^{\prime}, B^{\prime}, A^{\prime \prime}, B^{\prime \prime}$ appear in this order on $S$ (possibly $B^{\prime}=A^{\prime \prime}$ ). Let $\left|C \cap D_{1}\right|=\alpha \geq 0,\left|\operatorname{arc}_{C}\left(B^{\prime} A^{\prime \prime}\right)\right|=\beta \geq 0,\left|C \cap D_{2}\right|=\gamma \geq 0$. It follows from Observation 2.3 that $\alpha+\beta+\gamma<\pi$.

Draw the ray $L_{1}$ tangent to $C_{1} \cap D$ at $B^{\prime}$, and the ray $L_{2}$ tangent to $C_{2} \cap D$ at $A^{\prime \prime}$. Since $2 \alpha+\beta+2 \gamma<2 \pi$, by Lemma 3.6, these rays do not cross inside $D$, and they separate $D \cap D_{1}$ from $D \cap D_{2}$. If $C_{1}$ and $C_{2}$ touch at $B^{\prime}$, then $L_{1}$ and $L_{2}$ coincide. In this case $\beta=0$ and $\alpha+\gamma=\pi$ contradicting the assumption that $\alpha+\beta+\gamma<\pi$.

Therefore, $C_{1}$ and $C_{2}$ cross, and since $D \cap D_{1}$ is separated by a line from $D \cap D_{2}$, we conclude that $D_{1} \cap D_{2} \nsubseteq \subseteq D$.

If the arcs $C \cap D_{1}$ and $C \cap D_{2}$ do have interior points in common, then clearly $D_{1} \cap D_{2} \nsubseteq D$.

From this point on we assume that every intersection point of circles in $\mathcal{C}$ has degree $\geq 3$.

Lemma 3.8. Let $C_{1}, C_{2} \in \mathcal{C}$ be two different circles, then either $D_{1} \cap D_{2}$ includes a lens or there is a circle which touches $C_{1}$ or $C_{2}$ at a point on the boundary of $D_{1} \cap D_{2}$.

Proof. Clearly, if $C_{1}$ and $C_{2}$ touch, then there is nothing to prove. Assume then that $C_{1}$ and $C_{2}$ cross and $C_{1} \cap C_{2}=\{A, B\}$. We assume that no circle in $\mathcal{C}$ touches $C_{1}$ or $C_{2}$ at a point on the boundary of $D_{1} \cap D_{2}$.

Let $G_{\mathcal{C}}^{\prime}$ be the planar subgraph of $G_{\mathcal{C}}$ which is induced by the vertices of $G_{\mathcal{C}}$ which are in $D_{1} \cap D_{2}$. We regard the complement of $D_{1} \cap D_{2}$ as the unbounded face of $G_{\mathcal{C}}^{\prime}$.

For every $k \geq 2$ let $u_{k}$ denote the number of vertices inside int $\left(D_{1} \cap D_{2}\right)$ through which exactly $k$ circles pass. Let $v_{k}$ denote the number of vertices, different from $A$ and $B$, on the boundary of $D_{1} \cap D_{2}$ through which exactly $k$ circles pass. Denote by $w_{k}$ the number of faces in $G_{\mathcal{C}}^{\prime}$ which have exactly $k$ edges.

Let $V, E, F$ denote the number of vertices, edges, and faces of $G_{\mathcal{C}}^{\prime}$, respectively. Then $V=2+\sum_{k \geq 2} u_{k}+v_{k}$ and $F=\sum_{k \geq 2} w_{k}$. Counting the number of edges in two different ways we get

$$
2 E=\sum_{k \geq 2} k w_{k}=3 F+\sum_{k \geq 2}(k-3) w_{k} .
$$

Since we assume that $D_{1} \cap D_{2}$ includes no lens, all faces of $G_{\mathcal{C}}^{\prime}$ have at least three edges. Note that the unbounded face has exactly $2+\sum_{k \geq 2} v_{k}$ edges. Therefore,

$$
\sum_{k \geq 2}(k-3) w_{k} \geq \sum_{k \geq 2} v_{k}-1 .
$$

Observe that the degree in $G_{\mathcal{C}}^{\prime}$ of a vertex counted by $u_{k}$ is $2 k$ and the degree of a vertex counted by $v_{k}$ is $k+1$. Let $\operatorname{deg}(A)$ and $\operatorname{deg}(B)$ denote the degrees in $G_{\mathcal{C}}^{\prime}$ of $A$ and 
$B$, respectively. Counting the sum of the degrees of all vertices in two different ways we obtain

$$
2 E=\operatorname{deg}(A)+\operatorname{deg}(B)+\sum_{k \geq 2} 2 k u_{k}+(k+1) v_{k} .
$$

By the Euler formula $V-E+F=2$, so that plugging in (4)-(6) we obtain

$$
\begin{aligned}
-12= & -6 V+6 E-6 F=-6 V+2 E+2(2 E-3 F) \\
= & -6\left(2+\sum_{k \geq 2} u_{k}+v_{k}\right)+\operatorname{deg}(A)+\operatorname{deg}(B) \\
& +\sum_{k \geq 2}\left(2 k u_{k}+(k+1) v_{k}\right)+2 \sum_{k \geq 2}(k-3) w_{k} \\
= & -12+\operatorname{deg}(A)+\operatorname{deg}(B)+\sum_{k \geq 2}\left((2 k-6) u_{k}+(k-5) v_{k}\right) \\
& +2 \sum_{k \geq 2}(k-3) w_{k} \\
\geq & -12+\operatorname{deg}(A)+\operatorname{deg}(B)+\sum_{k \geq 2}\left((2 k-6) u_{k}+(k-5) v_{k}\right) \\
& +2\left(\sum_{k \geq 2} v_{k}-1\right) \\
= & -14+\operatorname{deg}(A)+\operatorname{deg}(B)+\sum_{k \geq 2}\left((2 k-6) u_{k}+(k-3) v_{k}\right) .
\end{aligned}
$$

Rearranging, we get

$$
\begin{aligned}
2 u_{2}+v_{2} & \geq-2+\operatorname{deg}(A)+\operatorname{deg}(B)+\sum_{k \geq 3}\left((2 k-6) u_{k}+(k-3) v_{k}\right) \\
& \geq-2+\operatorname{deg}(A)+\operatorname{deg}(B) \geq 2 .
\end{aligned}
$$

The last inequality is true because $\operatorname{deg}(A), \operatorname{deg}(B) \geq 2$. Every vertex counted by $u_{2}$ is an intersection point of precisely two circles in $\mathcal{C}$. Moreover, since we assume that no circle in $\mathcal{C}$ touches $C_{1}$ or $C_{2}$ at a point on the boundary of $D_{1} \cap D_{2}$, every vertex counted by $v_{2}$ is an intersection point of precisely two circles in $\mathcal{C}$. We assume that through every intersection point in $\mathcal{A}(\mathcal{C})$ at least three circles pass and hence $u_{2}=v_{2}=0$. In view of (7), this is a contradiction.

Corollary 3.9. Assume that $C_{1}, C_{2} \in \mathcal{C}$ and each of $C_{1}, C_{2}$ supports the longest arc of a lune, then either $D_{1} \cap D_{2}$ is a proper lens or $C_{1}$ and $C_{2}$ touch.

Proof. By Lemma 3.8, either $D_{1} \cap D_{2}$ includes a lens $l$ or it includes a tangency point $P$ of two circles $C_{3}, C_{4} \in \mathcal{C}$. In the first case, using Lemma 3.7, $l$ is supported by $C_{1}$ as well as by $C_{2}$ and hence $l=D_{1} \cap D_{2}$. In the second case since $P \in D_{1}$, by the second part of Lemma 3.7, $C_{1}$ is one of $C_{3}, C_{4}$, and similarly $C_{2}$ is one of $C_{3}, C_{4}$. Hence, $C_{1}$ and $C_{2}$ touch at $P$. 
Corollary 3.10. There are at most three lunes in $\mathcal{A}(\mathcal{C})$.

Proof. Assume $C_{1}, C_{2}, C_{3}, C_{4} \in \mathcal{C}$ and each of them supports the longest arc of some lune. By Corollary 3.9, for every $1 \leq i<j \leq 4$, either $D_{i} \cap D_{j}$ is a proper lens or $C_{i}$ and $C_{j}$ touch.

Note that if $C_{i}$ and $C_{j}$ touch at a point $P$ and $C_{k}$ also passes through $P$, then, by the second part of Lemma 3.7, $C_{k}$ coincides with $C_{i}$ or $C_{j}$. This means that all six intersections $D_{i} \cap D_{j}$ are lenses which contradicts Lemma 3.4.

\section{Proof of the Main Theorem}

Proof of Theorem 1.1. Assume to the contrary that every intersection point $P \in \mathcal{A}(\mathcal{C})$ has $d(P) \geq 3$.

Denote by $m$ the number of lenses and by $l$ the number of lunes. The faces of $G_{\mathcal{C}}$ counted by $f_{2}$ are exactly the proper lenses and the lunes. Therefore $f_{2} \leq m+l$. Let $u$ denote the number of edges of the unbounded face of $\mathcal{A}(\mathcal{C})$. We claim that $m \leq u$. Indeed, let $C_{1}, \ldots, C_{s}$ be all the circles in $\mathcal{C}$ that support a lens. By Lemma 3.2, the unbounded face has at least $u \geq s$ edges. By Lemma 3.4, there are at most $m \leq s$ lenses.

Substituting this in (1) we get

$$
t_{2}=6+\sum_{k \geq 3}(k-3) t_{k}+\sum_{k \geq 3}(k-3) f_{k}-f_{2} \geq 6+(m-3)-(m+l)=3-l,
$$

where the inequality is true because $f_{2} \leq m+l, \sum_{k \geq 3}(k-3) t_{k} \geq 0$, and $\sum_{k \geq 3}(k-3) f_{k} \geq$ $u-3 \geq m-3$, due to the unbounded face.

Since we assume $t_{2}=0$ we must have $l=3$ (because of Corollary 3.10). Note also that all inequalities must be equalities. Hence we deduce the following equalities: $f_{2}=m+l$, implying that all lenses are proper lenses. $\sum_{k \geq 3}(k-3) t_{k}=0$, implying that every intersection point has degree $3 . \sum_{k \geq 3}(k-3) f_{k}=m-3$, implying that the unbounded face has exactly $m$ edges. Hence (following our argument showing that $u \geq m$ ) every circle which includes an edge of the unbounded face must support a lens. Moreover, every bounded face which is neither a lens nor a lune must be a triangle (that is, a face with just three edges). An important corollary is that no two circles in $\mathcal{C}$ touch. For if two circles in $\mathcal{C}$ touch at a point $P$, then $d(P)=3$ implies that $P$ is a lens which is not a proper lens and we obtain a contradiction. From now on we assume that every two circles in $\mathcal{C}$ cross.

Let $C_{1}, C_{2}, C_{3}$ be the three circles which support the longest arcs of the three lunes in $\mathcal{A}(\mathcal{C})$. By Corollary 3.9, for every $1 \leq i<j \leq 3 D_{i} \cap D_{j}$ is a proper lens. Denote $l_{1}=D_{2} \cap D_{3}, l_{2}=D_{1} \cap D_{3}, l_{3}=D_{1} \cap D_{2}$.

Claim 4.1. Let $C \in \mathcal{C}$. Then $D$ includes at least one of $l_{1}, l_{2}, l_{3}$.

Proof. If $C$ is one of $C_{1}, C_{2}, C_{3}$, then clearly $D$ includes two of $l_{1}, l_{2}, l_{3}$. Let $C \in$ $\mathcal{C} \backslash\left\{C_{1}, C_{2}, C_{3}\right\}$ and assume that $D$ does not include any of $l_{1}, l_{2}, l_{3}$. Hence, $D$ is disjoint from the interior of each of $l_{1}, l_{2}, l_{3}$. 
$D \cap$ int $l_{2}=\emptyset$ implies that int $D \cap$ int $D_{1} \cap$ int $D_{3}=\emptyset$. It follows that $D_{3} \cap \operatorname{int}(D \cap$ $\left.D_{1}\right)=\emptyset$. Similarly, $D \cap \operatorname{int} l_{3}=\emptyset$ implies $D_{2} \cap \operatorname{int}\left(D \cap D_{1}\right)=\emptyset$. In other words, if we restrict our attention to the subfamily $\mathcal{C}^{\prime}=\left\{C, C_{1}, C_{2}, C_{3}\right\}$ then $D \cap D_{1}$ is a lens.

In the same way we obtain that also $D \cap D_{2}$ and $D \cap D_{3}$ are lenses (within $\mathcal{A}\left(\mathcal{C}^{\prime}\right)$ ). This contradicts Lemma 3.4.

$D_{1} \cap D_{2} \cap D_{3}$ has an empty interior so it is either empty or a single point.

Case 1: $D_{1} \cap D_{2} \cap D_{3}$ is a point $P$. Let $C \in \mathcal{C} \backslash\left\{C_{1}, C_{2}, C_{3}\right\}$. By Claim 4.1, $D$ must include at least one of $l_{1}, l_{2}, l_{3}$. Hence $P \in D$. We claim that $P \in \operatorname{int} D$. Assume to the contrary that $P \in C$. Then $D$ includes either one or two lenses of $l_{1}, l_{2}, l_{3}$. If $D$ includes just one lens, say $l_{1}$, then it must be disjoint from the interiors of $l_{2}$ and $l_{3}$. This is possible only if $C$ touches $C_{1}$ at $P$, which is impossible since every two circles in $\mathcal{C}$ cross. If $D$ includes two lenses, say $l_{2}$ and $l_{3}$, and is disjoint from the interior of the third, $l_{1}$, then it is easily seen by inspection that $C$ must coincide with $C_{1}$, contradicting our assumption on $C$.

Hence $P \in \operatorname{int} D$. It follows now that $D$ includes all lenses $l_{1}, l_{2}, l_{3}$. Denote the remaining intersection points of the circles $C_{1}, C_{2}$, and $C_{3}$ by $P_{1}, P_{2}, P_{3}$ (see Fig. 1). We regard the affine plane as the two-dimensional vector space $\mathrm{R}^{2}$ with $P$ as its origin. Then $P_{1}=O_{2}+O_{3}, P_{2}=O_{1}+O_{3}$, and $P_{3}=O_{1}+O_{2}$.

Therefore the distance of each of $P_{1}, P_{2}$, and $P_{3}$ to the point $O_{1}+O_{2}+O_{3}$ is 1 , which shows that the circle through $P_{1}, P_{2}$, and $P_{3}$ is a unit circle. Moreover,

$$
\Delta\left(P_{1} P_{2} P_{3}\right)=\left(O_{1}+O_{2}+O_{3}\right)-\Delta\left(O_{1} O_{2} O_{3}\right) .
$$

Hence, $\Delta\left(P_{1} P_{2} P_{3}\right)$ is congruent to $\Delta\left(O_{1} O_{2} O_{3}\right)$, which is an acute triangle by Lemma 3.1. It follows that the circumcircle of $\Delta\left(P_{1} P_{2} P_{3}\right)$ is a unit circle which we denote by $S$.

Let $C \in \mathcal{C} \backslash\left\{C_{1}, C_{2}, C_{3}\right\}$. $D$ includes $l_{1}, l_{2}$, and $l_{3}$, and so in particular $D \supset \Delta\left(P_{1} P_{2} P_{3}\right)$. It follows that $C=S$.

In other words $\#(\mathcal{C}) \leq 4$. If $\mathcal{C}=\left\{S, C_{1}, C_{2}, C_{3}\right\}$ then $\mathcal{C}$ is exceptional. Otherwise, $\mathcal{C}=\left\{C_{1}, C_{2}, C_{3}\right\}$ and each of $P_{1}, P_{2}$, and $P_{3}$ has degree 2 .

Case 2: $D_{1} \cap D_{2} \cap D_{3}=\emptyset$. Denote by $B_{1}, B_{2}, B_{3}$ and $A_{1}, A_{2}, A_{3}$ the intersection points of $C_{1}, C_{2}$, and $C_{3}$ as indicated in Fig. 6 .

Lemma 4.2. Let $C, C_{1}, C_{2}$ be three pairwise crossing unit circles. If $D \supset D_{1} \cap D_{2}$, then $\measuredangle O_{1} O O_{2}>\pi / 2$.

Proof. Denote by $A, B$ the intersection points of $C_{1}$ and $C_{2}$. Let $S_{A}, S_{B}$ be the unit disks centered at $A, B$, respectively. Note that $O \in S_{A} \cap S_{B}$. Observe that the boundaries of $S_{A}$ and $S_{B}$ intersect at $O_{1}$ and $O_{2} . S_{A} \cap S_{B}$ is included in the disk whose diameter is $\left[O_{1} O_{2}\right]$. Hence $\measuredangle O_{1} O O_{2}>\pi / 2$.

Claim 4.3. Assume $C \in \mathcal{C}$ and $D$ includes $l_{1}$ but not $l_{2}$ or $l_{3}$. Then $C$ intersects $C_{1}$ in two points on $\operatorname{arc}_{C_{1}}\left(B_{2} B_{3}\right)$.

Remark. By symmetry, Claim 4.3 is true if we change the role of $l_{1}$ by $l_{2}$ or $l_{3}$, and, correspondently, $C_{1}$ by $C_{2}$ or $C_{3}$. 


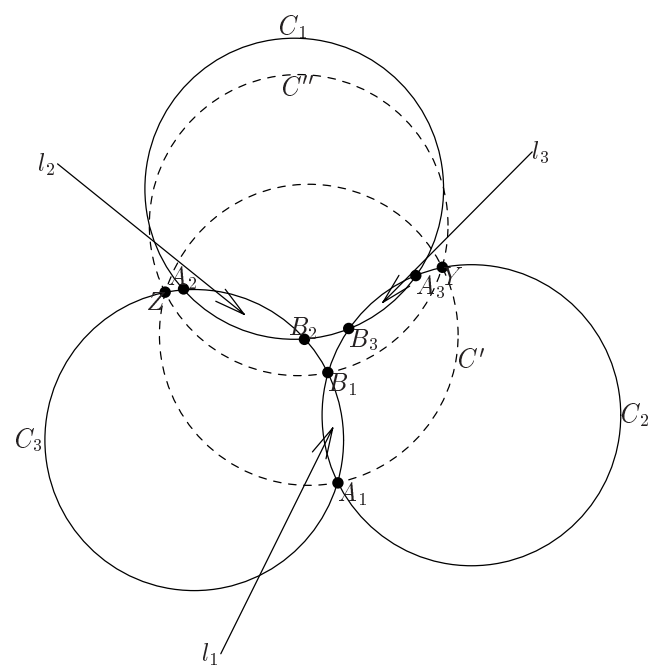

Fig. 6. Case 2.

Proof. We show that $O$ is inside $\angle O_{2} O_{1} O_{3} . D$ is disjoint from int $l_{2}=\operatorname{int}\left(D_{1} \cap D_{3}\right)$ and from int $l_{3}=\operatorname{int}\left(D_{1} \cap D_{2}\right)$. Therefore, by Lemma 3.1, the triangles $\Delta\left(O_{1} O_{2} O\right)$ and $\triangle\left(\mathrm{O}_{1} \mathrm{O}_{3} \mathrm{O}\right)$ are acute triangles. Moreover, by Lemma 3.1, all the angles of $\triangle\left(\mathrm{O}_{1} \mathrm{O}_{2} \mathrm{O}_{3}\right)$ are acute. If $O \notin \angle O_{2} O_{1} O_{3}$, then either $O \in \angle O_{1} O_{3} O_{2}$ or $O \in \angle O_{1} O_{2} O_{3}$. We assume, without loss of generality, that $O \in \angle O_{1} O_{3} O_{2}$. Since $O \notin \angle O_{2} O_{1} O_{3}$, it follows that $O_{3} \in \angle O_{1} O O_{2}$. By Lemma 4.2, $\measuredangle O_{2} O O_{3}>\pi / 2$. We obtain a contradiction since now $\measuredangle O_{2} O O_{3} \leq \measuredangle O_{2} O O_{1}<\pi / 2$.

We conclude that $\overrightarrow{O_{1} O}$ must be between $\overrightarrow{O_{1} O_{2}}$ and $\overrightarrow{O_{1} O_{3}}$. Since $D$ is disjoint from int $l_{2}$ and int $l_{3}, D \cap C_{1}$ must be disjoint from the relative interiors of both arcs $D_{2} \cap C_{1}$ and $D_{3} \cap C_{1}$. The center of the arc $C_{1} \cap D$ is between the centers of $C_{1} \cap D_{2}$ and $C_{1} \cap D_{3}$. It follows now that $D \cap C_{1}$ must be included in $\operatorname{arc}_{C_{1}}\left(B_{2} B_{3}\right)$.

Claim 4.4. Assume that $C \in \mathcal{C} \backslash\left\{C_{1}, C_{2}, C_{3}\right\}$. If $C$ supports a lens, then $D$ includes exactly one of the lenses $l_{1}, l_{2}$, or $l_{3}$.

Proof. By Claim 4.1, $D$ includes at least one of $l_{1}, l_{2}$, or $l_{3}$. Assume, without loss of generality, that $D \supset l_{1} \cup l_{2}$ and that $D \cap D^{\prime}$ is a lens. $D$ includes also $\operatorname{arc}_{C_{3}}\left(B_{1} B_{2}\right) . D^{\prime}$ must include only $l_{3}$ (and not any of $l_{1}, l_{2}$ ) for otherwise $D \cap D^{\prime}$ includes either $l_{1}$ or $l_{2}$ and therefore is not a lens.

By Claim 4.3, int $D^{\prime}$ meets the relative interior of $\operatorname{arc}_{C_{3}}\left(B_{1} B_{2}\right)$, implying that int $(D \cap$ $\left.D^{\prime}\right)$ also meets it. This is contradicting the assumption that $D \cap D^{\prime}$ is a lens.

Claim 4.5. Each of $A_{1}, A_{2}$, and $A_{3}$ is included in the interior of some unit disk bounded by a circle from $\mathcal{C}$. 
Proof. We prove the claim for $A_{1}$. Assume that the claim is not true for $A_{1}$. Let $C_{2}^{\prime} \in \mathcal{C}$ be the circle so that $D_{2} \backslash$ int $D_{2}^{\prime}$ is a lune. Since $A_{1} \in D_{2}^{\prime}, A_{1} \notin$ int $D_{2}^{\prime}$ implies that $C_{2}^{\prime}$ passes through $A_{1}$. Similarly, let $C_{3}^{\prime} \in \mathcal{C}$ be the circle so that $D_{3} \backslash$ int $D_{3}^{\prime}$ is a lune, then $C_{3}^{\prime}$ passes through $A_{1}$. However, $d\left(A_{1}\right)=3$ and $C_{2}, C_{3}$ pass through $A_{1}$. It follows that $C_{2}^{\prime}=C_{3}^{\prime}$. Denote $C^{\prime}=C_{2}^{\prime}=C_{3}^{\prime}$. $D^{\prime}$ includes all lenses $l_{1}, l_{2}, l_{3}$. Denote the intersection points of $C^{\prime}$ with $C_{2}$ and $C_{3}$ other than $A_{1}$ by $Y, Z$, respectively (see Fig. 6).

The face whose two edges $\operatorname{are~}_{\operatorname{arc}_{C_{3}}}\left(A_{1} B_{1}\right)$ and $\operatorname{arc}_{C^{\prime}}\left(A_{1} Y\right)$ must have exactly three edges so there must be a circle (other than $C_{2}$ ) in $\mathcal{C}$ which passes through $Y$ and $B_{1}$. Similarly, there must be a circle (other than $C_{3}$ ) in $\mathcal{C}$ which passes through $Z$ and $B_{1}$. Since $d\left(B_{1}\right)=3$ and $C_{2}$ and $C_{3}$ pass through $B_{1}$, it follows that the same circle $C^{\prime \prime} \in \mathcal{C}$ passes through $Y, B_{1}$, and $Z$.

Observe that $Y \neq A_{3}$ for otherwise there would be four circles through this point, namely $C_{1}, C_{2}, C^{\prime}$, and $C^{\prime \prime}$. It is easily seen now that the face whose edge is $\operatorname{arc}_{C^{\prime \prime}}\left(Y B_{1}\right)$ which is adjacent to the face $A_{1} Y B_{1}$ has more than three edges (as no other circles pass through $Y$ nor $B_{1}$ ), a contradiction.

Let $\Delta_{0}$ denote the closed region which is bounded by the $\operatorname{arcs} \operatorname{arc}_{C_{1}}\left(B_{2} B_{3}\right), \operatorname{arc}_{C_{2}}$ $\left(B_{3} B_{1}\right)$, and $\operatorname{arc}_{C_{3}}\left(B_{1} B_{2}\right)$.

Lemma 4.6. Let $C \in \mathcal{C}$. Then $D \cap \Delta_{0} \cap \overline{B_{1} A_{1}} \neq \emptyset$. (Similarly for $\overline{B_{2} A_{2}}$ and $\overline{B_{3} A_{3}}$.)

Proof. If $D \supset l_{1}$, then clearly $B_{1} \in D \cap \Delta_{0} \cap \overline{B_{1} A_{1}}$. If $D \supset\left(l_{2} \cup l_{3}\right)$, then $D$ includes $\operatorname{arc}_{C_{1}}\left(B_{2} B_{3}\right)$. Therefore, $D \cap \Delta_{0} \cap \overline{B_{1} A_{1}}$ includes the intersection point of $\operatorname{arc}_{C_{1}}\left(B_{2} B_{3}\right)$ with $\overline{B_{1} A_{1}}$ (indeed, this intersection point exists because $\overline{B_{1} A_{1}}$ separates $l_{2}$ and $l_{3}$ ).

The only case which is left to check is where $D$ includes only one of $l_{2}$ or $l_{3}$, and not $l_{1}$. Assume, without loss of generality, that $D$ includes $l_{3}$ and not $l_{1}$ or $l_{2}$. By Claim 4.3, $D$ includes a subarc of $\operatorname{arc}_{C_{3}}\left(B_{1} B_{2}\right)$. Since $D$ is convex, $D$ includes an interval connecting $B_{3}$ to a point on $\operatorname{arc}_{C_{3}}\left(B_{1} B_{2}\right)$. This interval intersects $\overline{B_{1} A_{1}}$ inside $\Delta_{0}$.

Claim 4.7. For each of the lenses $l_{1}, l_{2}$, and $l_{3}$ there is a circle $C \in \mathcal{C}$ such that $D$ includes that lens and not the two others.

Proof. We prove the claim for $l_{1}$. Let $Q$ be the intersection point of a circle from $\mathcal{C}$ and the ray $\overrightarrow{B_{1} A_{1}}$, which has maximum distance from $B_{1}$. One of the circles $C$ through $Q$ includes an edge of the unbounded face. By Claim 4.5, $Q \neq A_{1}$. Consequently, $C$ is not one of $C_{1}, C_{2}, C_{3}$. We show that $D$ includes $l_{1}$ and not any of $l_{2}$ or $l_{3}$.

By Lemma 4.6, $D \cap \Delta_{0} \cap \overline{B_{1} A_{1}} \neq \emptyset$. It follows, from the convexity of $D$, that $D \supset\left[B_{1} A_{1}\right]$. Hence $D \supset l_{1}$.

Observe that if $D$ includes $l_{2}$ or $l_{3}$, then, by Claim 4.4, $C$ does not support a lens. This is a contradiction because $C$ includes an edge of the unbounded face.

We will need the following geometric observation in what follows.

Lemma 4.8. Let $C, C_{1}, C_{2}$ be three distinct unit circles. Assume that $C_{1}$ and $C_{2}$ cross, and $D \supset D_{1} \cap D_{2}$. Denote the intersection points of $C_{1}$ and $C_{2}$ by $M, N$. Denote the 


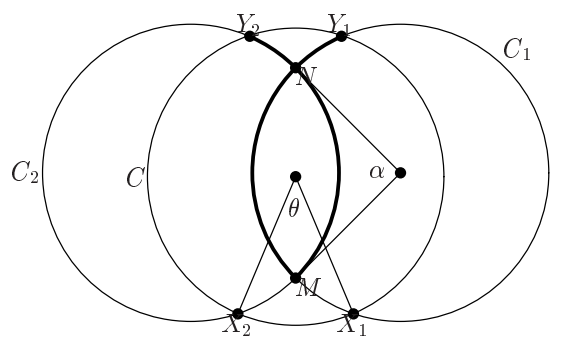

Fig. 7. Lemma 4.8 .

intersection points of $C$ and $C_{1}$ by $X_{1}, Y_{1}$ and those of $C$ and $C_{2}$ by $X_{2}, Y_{2}$ (as indicated in Fig. 7). Let $\theta=\left|\operatorname{arc}_{C}\left(X_{1} X_{2}\right)\right|$ and let $\alpha=\left|\operatorname{arc}_{C_{1}}(M N)\right|$. Then

$$
\left|\operatorname{arc}_{C_{1}}\left(Y_{1} M\right)\right|+\left|\operatorname{arc}_{C_{2}}\left(Y_{2} M\right)\right|=\pi+\alpha-\theta .
$$

Proof. Rotate (and if necessary flip) the plane so that $\overline{O_{1} O_{2}}$ is horizontal, $O_{1}$ is to the right of $O_{2}$, and $X_{1}, X_{2}$ are below $\overline{O_{1} O_{2}}$. We show how to deal with the case where $O$ is on or above $\overline{O_{1} O_{2}}$. The other case can be treated similarly. Note that each of the quadrilaterals $O X_{1} O_{1} Y_{1}$ and $O X_{2} O_{2} Y_{2}$ is a rhombus.

$$
\begin{aligned}
\left|\operatorname{arc}_{C_{2}}\left(M Y_{2}\right)\right|+\left|\operatorname{arc}_{C_{1}}\left(M Y_{1}\right)\right| & =\alpha+\measuredangle O_{1} O_{2} Y_{2}+\measuredangle O_{2} O_{1} Y_{1} \\
& =\alpha+\measuredangle O_{1} O_{2} O+\measuredangle O O_{2} Y_{2}+\measuredangle O_{2} O_{1} O+\measuredangle O O_{1} Y_{1} \\
& =\alpha+\measuredangle O_{1} O_{2} O+\measuredangle O_{2} O X_{2}+\measuredangle O_{2} O_{1} O+\measuredangle O_{1} O X_{1} \\
& =\alpha+\measuredangle O_{1} O_{2} O+\measuredangle O_{2} O_{1} O+\measuredangle O_{2} O O_{1}-\theta \\
& =\pi+\alpha-\theta .
\end{aligned}
$$

Denote by $\alpha_{1}, \alpha_{2}, \alpha_{3}$ the lengths of the arcs $C_{1} \cap D_{2}, C_{2} \cap D_{3}$, and $C_{3} \cap D_{1}$, respectively. Denote by $\theta_{1}, \theta_{2}, \theta_{3}$ the lengths of $\operatorname{arc}_{C_{1}}\left(B_{2} B_{3}\right)$, $\operatorname{arc}_{C_{2}}\left(B_{3} B_{1}\right)$, and $\operatorname{arc}_{C_{3}}\left(B_{1} B_{2}\right)$, respectively. By reducing to the extreme case where $C_{1}, C_{2}$, and $C_{3}$ are pairwise touching we observe that for every $1 \leq i \leq 3, \theta_{i}<\pi / 3$. For $i=1,2,3$ let $C_{i}^{\prime} \in \mathcal{C}$ be a circle which is guaranteed by Claim 4.7 , for $l_{i}$. Thus, $D_{i}^{\prime}$ includes $l_{i}$ alone from $l_{1}, l_{2}$, and $l_{3}$.

$D_{1}^{\prime}$ includes $l_{1}$ and is disjoint from the interiors of both $l_{2}$ and $l_{3}$. Therefore $C_{1}^{\prime}$ separates the interior of $l_{1}$ from the interior of $l_{3}$.

Let $F_{12}=C_{1}^{\prime} \cap \operatorname{arc}_{C_{2}}\left(B_{3} B_{1}\right)$, and similarly define $F_{13}, F_{21}, F_{23}, F_{31}$, and $F_{32}$ (see Fig. 8).

Also let $\theta_{1}^{\prime}=\left|\operatorname{arc}_{C_{1}^{\prime}}\left(F_{12} F_{13}\right)\right|, \theta_{2}^{\prime}=\left|\operatorname{arc}_{C_{2}^{\prime}}\left(F_{21} F_{23}\right)\right|$, and $\theta_{3}^{\prime}=\left|\operatorname{arc}_{C_{3}^{\prime}}\left(F_{31} F_{32}\right)\right|$.

Claim 4.9. $\theta_{1}^{\prime}, \theta_{2}^{\prime}, \theta_{3}^{\prime} \leq \max \left(\theta_{1}, \theta_{2}, \theta_{3}\right)<\pi / 3$.

Proof. We prove the claim for $\theta_{1}^{\prime}=\left|\operatorname{arc}_{C_{1}^{\prime}}\left(F_{12} F_{13}\right)\right|$. The length of $\operatorname{arc}_{C_{1}^{\prime}}\left(F_{12} F_{13}\right)$ depends monotonously on the length of the interval $\left[F_{12} F_{13}\right]$. Observe that $\left[F_{12} F_{13}\right]$ is included in $\Delta\left(B_{1} B_{2} B_{3}\right)$ and therefore the length of $\left[F_{12} F_{13}\right]$ is less than or equal to the 


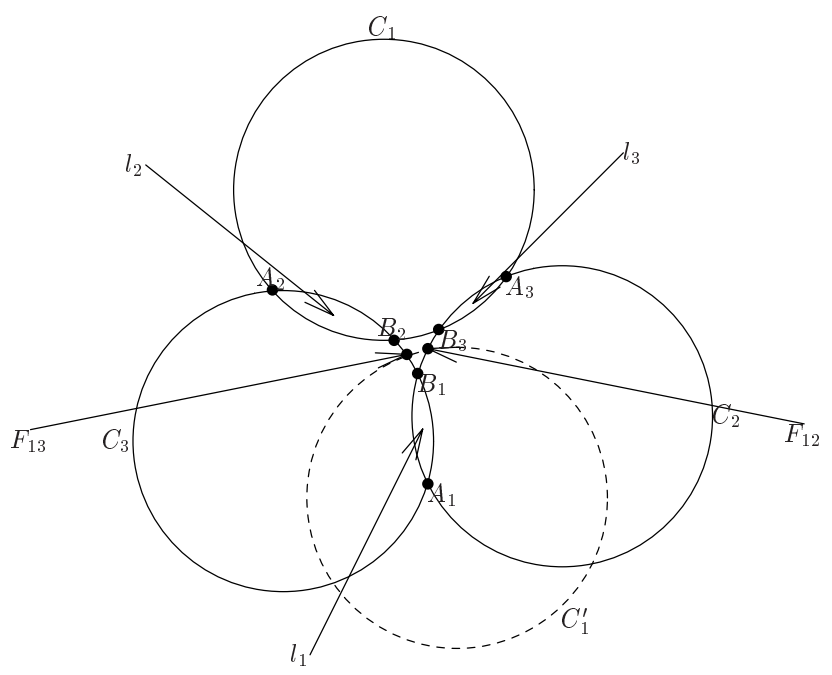

Fig. 8. Claim 4.9.

maximum of the lengths of the intervals $\left[B_{3} B_{1}\right],\left[B_{1} B_{2}\right]$, and $\left[B_{2} B_{3}\right]$. Consequently, the length of $\operatorname{arc}_{C_{1}^{\prime}}\left(F_{12} F_{13}\right)$ is at $\operatorname{most} \max \left(\theta_{1}, \theta_{2}, \theta_{3}\right)$.

Let $F_{12}^{\prime}$ denote the second intersection point of $C_{1}^{\prime}$ and $C_{2}$ (other than $F_{12}$ ). Similarly define $F_{13}^{\prime}, F_{21}^{\prime}, F_{23}^{\prime}, F_{31}^{\prime}$, and $F_{32}^{\prime}$.

$D_{1}^{\prime} \supset D_{2} \cap D_{3}$. Therefore, using Lemma 4.8 with $C_{1}^{\prime}, C_{2}$, and $C_{3}$, We obtain

$$
\left|\operatorname{arc}_{C_{2}}\left(B_{1} F_{12}^{\prime}\right)\right|+\left|\operatorname{arc}_{C_{3}}\left(B_{1} F_{13}^{\prime}\right)\right|=\pi+\alpha_{1}-\theta_{1}^{\prime}>\frac{2 \pi}{3}+\alpha_{1}
$$

Similarly, using Lemma 4.8 twice more, once with $C_{2}^{\prime}, C_{1}, C_{3}$ and once with $C_{3}^{\prime}, C_{1}$, $C_{2}$, we obtain

$$
\begin{aligned}
& \left|\operatorname{arc}_{C_{1}}\left(B_{2} F_{21}^{\prime}\right)\right|+\left|\operatorname{arc}_{C_{3}}\left(B_{2} F_{23}^{\prime}\right)\right|=\pi+\alpha_{2}-\theta_{2}^{\prime}>\frac{2 \pi}{3}+\alpha_{2}, \\
& \left|\operatorname{arc}_{C_{1}}\left(B_{3} F_{31}^{\prime}\right)\right|+\left|\operatorname{arc}_{C_{2}}\left(B_{3} F_{32}^{\prime}\right)\right|=\pi+\alpha_{3}-\theta_{3}^{\prime}>\frac{2 \pi}{3}+\alpha_{3} .
\end{aligned}
$$

Since $C_{1}$ supports the longest arc of a lune, $\operatorname{arc}_{C_{1}}\left(B_{2} F_{21}^{\prime}\right), \operatorname{arc}_{C_{1}}\left(B_{3} F_{31}^{\prime}\right)$, and $\operatorname{arc}_{C_{1}}\left(B_{2} B_{3}\right)$ are included in an open semicircle on $C_{1}$. Therefore,

$$
\left|\operatorname{arc}_{C_{1}}\left(B_{2} F_{21}^{\prime}\right)\right|+\left|\operatorname{arc}_{C_{1}}\left(B_{3} F_{31}^{\prime}\right)\right|+\left|\operatorname{arc}_{C_{1}}\left(B_{2} B_{3}\right)\right|<\pi
$$

A similar argument for $C_{2}$ and $C_{3}$ gives

$$
\begin{aligned}
& \left|\operatorname{arc}_{C_{2}}\left(B_{3} F_{32}^{\prime}\right)\right|+\left|\operatorname{arc}_{C_{2}}\left(B_{1} F_{12}^{\prime}\right)\right|+\left|\operatorname{arc}_{C_{2}}\left(B_{3} B_{1}\right)\right|<\pi, \\
& \left|\operatorname{arc}_{C_{3}}\left(B_{1} F_{13}^{\prime}\right)\right|+\left|\operatorname{arc}_{C_{3}}\left(B_{2} F_{23}^{\prime}\right)\right|+\left|\operatorname{arc}_{C_{3}}\left(B_{1} B_{2}\right)\right|<\pi .
\end{aligned}
$$


Summing up (11)-(13), we get

$$
\begin{aligned}
3 \pi> & \left(\left|\operatorname{arc}_{C_{2}}\left(B_{1} F_{12}^{\prime}\right)\right|+\left|\operatorname{arc}_{C_{3}}\left(B_{1} F_{13}^{\prime}\right)\right|\right) \\
& +\left(\left|\operatorname{arc}_{C_{1}}\left(B_{2} F_{21}^{\prime}\right)\right|+\left|\operatorname{arc}_{C_{3}}\left(B_{2} F_{23}^{\prime}\right)\right|\right) \\
& +\left(\left|\operatorname{arc}_{C_{1}}\left(B_{3} F_{31}^{\prime}\right)\right|+\left|\operatorname{arc}_{C_{2}}\left(B_{3} F_{32}^{\prime}\right)\right|\right) \\
& +\left(\theta_{1}+\theta_{2}+\theta_{3}\right) \\
> & 2 \pi+\alpha_{1}+\alpha_{2}+\alpha_{3}+\theta_{1}+\theta_{2}+\theta_{3}=3 \pi,
\end{aligned}
$$

by (8)-(10) and since $\alpha_{1}+\alpha_{2}+\alpha_{3}+\theta_{1}+\theta_{2}+\theta_{3}$ is the sum of the internal angles of $\triangle\left(\mathrm{O}_{1} \mathrm{O}_{2} \mathrm{O}_{3}\right)$. We thus reached the desired contradiction.

\section{Concluding Remarks}

It is interesting to note that Theorem 1.1 remains true if $\mathcal{C}$ is a family of (at least five) pairwise intersecting translates of the boundary of a centrally symmetric convex body. The proof goes through the same lines as the proof presented in this paper. Clearly, one cannot use the notion of an angle as it is used above any more. However, the main lemmata and the structure of the proof remain identical. When an angle whose apex is a center of some disk is measured, one should usually take the length of the corresponding arc on the boundary of that disk (assuming that all bodies are normalized so that the length of their boundaries is $2 \pi$ ).

We can state this result in the following form.

Theorem 5.1. Let $\|\cdot\|$ be a norm on $\mathrm{R}^{2}$, and let $A$ be a finite set of points with diameter $\leq 2$. Then there exist a point $p$ in the plane such that $\|p-a\|=1$ is true for precisely two elements $a \in A$.

As mentioned in the Introduction, Theorem 1.1 does not generalize to the case of circles with different radii. In [ALPS], however, we did manage to prove the following weaker form of a generalization of Theorem 1.1.

Theorem 5.2. $\quad$ Let $\mathcal{C}$ be a family of $n$ pairwise intersecting circles in the plane. If $n$ is sufficiently large and $\mathcal{C}$ is not a pencil, then there exists an intersection point incident to at most three circles.

In [NPPS] we generalized Theorem 5.2 to the case where $\mathcal{C}$ is a family of pseudocircles.

Finally, we already noted in the Introduction that the analogue of Theorem 1.1 to the case where $\mathcal{C}$ is a family of unit circles, which are not necessarily pairwise intersecting, is not valid. However, all known counterexamples to this case are quite sparse. In this context we conjecture the following.

Conjecture 5.3. Let $\mathcal{C}$ be a family of $n$ unit circles in a bounded region. If $n$ is large enough, then there exists an intersection point of degree 2. 


\section{Acknowledgments}

The author thanks Micha A. Perles for valuable remarks on the proof presented here. Many thanks to the referees for their efforts and very detailed reports.

\section{References}

[ALPS] N. Alon, H. Last, R. Pinchasi, and M. Sharir, On the complexity of arrangements of circles in the plane, Discrete Comput. Geom. 26 (2001), 465-492.

[BB] A. Bálintová and V. Bálint, On the number of circles determined by $n$ points in the Euclidean plane, Acta Math. Hungar. 63(3) (1994),(3), 283-289.

[B1] A. Bezdek, On the density of dual circle coverings, Geom. Dedicata 33 (1990), 227-238.

[B2] A. Bezdek, On the intersection points of unit circles, Amer. Math. Monthly 99 (1992), 779-780.

[B3] A. Bezdek, Incidence problems for points and unit circles, Paul Erdös and His Mathematics (A. Sali, M. Simonovits and V.T. Sós, eds.), J. Bolyai Mathematical Society, Budapest, 1999, pp. 33-36.

[B4] A. Bezdek, On unit circles which avoid all but two points of a given pointset, European J. Combin., to appear.

[BFT] A. Bezdek, F. Fodor, and I. Talata, A Sylvester-type theorem about circles, Discrete Math., to appear.

[BM] P. Borwein and W.O.J. Moser, A survey of Sylvester's problem and its generalizations, Aequations Math. 40 (1990), 111-135.

[G] T. Gallai, Solution of problem 4065, Amer. Math. Monthly 51 (1944), 169-171.

[M] T.S. Motzkin, The lines and planes connecting the points of a finite set, Trans. Amer. Math. Soc. 70 (1951), 451-464.

[NPPS] E. Nevo, J. Pach, R. Pinchasi, and M. Sharir, Lenses in arrangements of pseudodisks and their application, preprint.

[PA] J. Pach and P. K.Agarwal, Combinatorial Geometry, Wiley, New York, 1995

[S] J.J. Sylvester, Mathematical question 11851, Ed. Times 59 (1893), 98-99.

Received March 26, 2001, and in revised form December 11, 2001. Online publication October 29, 2002. 